\title{
PRODUTIVIDADE E ARMAZENAMENTO DE CEBOLA (Allium cepa L.) SUBMETIDA A DOSES DE NITROGÊNIO E POTÁSSIO VIA FERTIRRIGAÇÃO EM CULTIVO DE VERÃO
}

\author{
Yield and storage of onion (Allium cepa L.) submitted to nitrogen and potassium \\ levels through fertirrigation in summer planting
}

\author{
Geraldo Milanez de Resende ${ }^{1}$, Nivaldo Duarte Costa ${ }^{1}$
}

\begin{abstract}
RESUMO
O experimento foi conduzido no período de agosto de 2001 a março de 2002, com o objetivo de avaliar a influência de doses de nitrogênio $(\mathrm{N})$ e potássio $(\mathrm{K})$ aplicadas via fertirrigação sobre o rendimento e armazenamento da cebola. Utilizou-se a cultivar Alfa Tropical, no delineamento experimental de blocos ao acaso, em esquema fatorial 4 × 3 , compreendendo quatro doses de nitrogênio $(0$, $60,120$ e $180 \mathrm{~kg} / \mathrm{ha})$ e três doses de potássio $\left(0,90\right.$ e $180 \mathrm{~kg} / \mathrm{ha}$ de $\left.\mathrm{K}_{2} \mathrm{O}\right)$, com três repetições. A produtividade comercial apresentou efeito linear quando se aplicou $90,0 \mathrm{~kg} / \mathrm{ha}$ de $\mathrm{K}_{2} \mathrm{O}$ e efeitos quadráticos na ausência da adubação potássica com máxima produtividade na dose de 96,5 kg/ha e mínima produtividade na dose de 136,5 kg/ha de nitrogênio na dose de $180,0 \mathrm{~kg} / \mathrm{ha}$ de $\mathrm{K}_{2} \mathrm{O}$. O incremento das doses de $\mathrm{N}$ reduziu a produtividade não comercial (refugos) de forma linear. A massa fresca dos bulbos evidenciou efeitos lineares com o incremento das doses de N. Não se constataram efeitos significativos aos 40 dias, após cura, para perda de massa, evidenciando-se efeito significativo apenas aos 80 dias, após cura, com a aplicação de $\mathrm{N}$ e $\mathrm{K}$.
\end{abstract}

Termos para indexação: Allium cepa, nutrição, rendimento, conservação pós-colheita.

\section{ABSTRACT}

This study was carried out from August 2001 to March 2002 to evaluate the influence of nitrogen (N) and potassium (K) levels through fertirrigation on yield and post-harvest storage of onion bulbs. Cultivar Alfa Tropical was used in a randomized complete block design, in a $4 \times 3$ factorial arrangement, with four nitrogen levels $(0,60,120$, and $180 \mathrm{~kg} / \mathrm{ha})$ and three potassium levels $(0,90$, and 180 $\mathrm{kg} / \mathrm{ha} \mathrm{K}_{2} \mathrm{O}$ ) with three replications. The commercial yield showed linear effect in the doses of $90 \mathrm{~kg} / \mathrm{ha} \mathrm{K}_{2} \mathrm{O}$ and quadratic effect in the absence of potassium fertilization, with maximum yield with the level of $96.5 \mathrm{~kg} / \mathrm{ha}$ nitrogen and minimum yield with the level of 136.5 $\mathrm{kg} / \mathrm{ha}$ nitrogen with the dose of $180 \mathrm{~kg} / \mathrm{ha} \mathrm{K} \mathrm{O}$. The increment of the levels of $\mathrm{N}$ reduced the non-commercial yield (culls) in a linear way. Linear effects were observed with the increase of $\mathrm{N}$ doses for bulb fresh mass. There were no significant effects at 40 days of curing for mass loss, however, at 80 days of curing, significant effect was evidenced with the application of $\mathrm{N}$ and $\mathrm{K}$.

Index terms: Allium cepa, yield, nutrition, yield, post-harvest conservation.

(Recebido em 4 de julho de 2008 e aprovado em 19 de março de 2009)

\section{INTRODUÇÃo}

A produção mundial de cebola (Allium cepa L.), em 2006, foi de 61,64 milhões de toneladas, cultivadas em uma área 3,34 milhões de hectares, o que proporcionou uma produtividade média de 18,45 t/ha (FAO, 2008). No Brasil, a cebola ocupa o terceiro lugar em importância econômica (Souza \& Resende, 2002). A produtividade média nacional se situou em 21,26 t/ha, sendo que nos estados de Pernambuco e Bahia, maiores produtores do Nordeste, alcançou-se uma produtividade média é de 18,78 e 23,49 t/ ha, respectivamente (IBGE, 2008).

Os elementos mais absorvidos em termos de porcentagem na matéria seca da cebola são o potássio e nitrogênio (Pôrto et al., 2007). O nitrogênio influencia significativamente a produtividade da cebola, segundo diferentes autores. O diâmetro do bulbo e a produtividade aumentaram com o incremento das doses até $80 \mathrm{~kg} / \mathrm{ha}$ de $\mathrm{N}$, não havendo diferenças significativas da dose de 120 kg/ha de N (Singh \& Sharma, 1991). Farias \& Pereira (1992), verificaram nas condições do Vale do São Francisco que o nível econômico de $\mathrm{N}$ para produtividade na cultura da cebola é de $115 \mathrm{~kg} / \mathrm{ha}$. A aplicação de $150 \mathrm{~kg} / \mathrm{ha}$ de N aumentou a altura da planta, número de folhas por planta, peso e tamanho do bulbo e a produtividade, não havendo diferenças significativas da dose de $100 \mathrm{~kg} / \mathrm{ha}$ de N, para o peso do bulbo e a produtividade (Vachchani \& Patel, 1996), Enquanto melhores respostas em termos de produtividade foram obtidas com a dose de $263 \mathrm{~kg} / \mathrm{ha}$ de $\mathrm{N}$ (Boyhan et al., 2007). Entretanto, outros trabalhos não encontraram respostas positivas quando variaram as doses de $\mathrm{N}$ de 84

${ }^{1}$ Embrapa - Centro de Pesquisa Agropecuária do Trópico Semi-Árido, Caixa Postal 23, CEP 56302-970 Petrolina, PE - gmilanez@cpatsa.embrapa.br; ndcosta@cpatsa.embrapa.br 
a $224 \mathrm{~kg} / \mathrm{ha}$ (Batal et al., 1994) e de 318 a $480 \mathrm{~kg} / \mathrm{ha}$ de N (Vidigal, 2000).

Apesar da cebola extrair grandes quantidades de potássio, respostas da cultura a esse nutriente, de maneira geral, não têm sido observadas (Filgueira, 2008) e há poucos resultados que confirmam efeito significativo do fertilizante potássico na produtividade, embora não se conheça o seu efeito sobre a qualidade e a conservação do produto (Magalhães, 1993). Sharma (1992) e Shukla et al. (1992), informam um incremento na produtividade com a aplicação de $40 \mathrm{~kg} / \mathrm{ha}$ de potássio, não se verificando nenhum efeito adicional quando se aplicou dose superiores $(80$ e $160 \mathrm{~kg} /$ ha de $\mathrm{K}_{2} \mathrm{O}$, respectivamente). Aumento no diâmetro e na massa de matéria fresca do bulbo foi relatado por Mohanty \& Das (2001) com a aplicação de $60 \mathrm{~kg} /$ ha de $\mathrm{K}_{2} \mathrm{O}$, enquanto Akhtar et al. (2002) observaram aumento na produtividade até a dose de $200 \mathrm{~kg} / \mathrm{ha}$ de $\mathrm{K}_{2} \mathrm{O}$, quando em presença de adubações nitrogenadas e fosfatadas.

No que se refere a bulbos armazenados sob condições ambientais, Singh \& Dhankhar (1991), verificaram que a perda de peso e a incidência de bulbos podres e brotados aumentaram com o incremento das doses de nitrogênio ( 80 a $160 \mathrm{~kg} / \mathrm{ha}$ de $\mathrm{N}$ ) e reduziram com a dose de $100 \mathrm{~kg} / \mathrm{ha}$ de $\mathrm{K}_{2} \mathrm{O}$. As doses de $80 \mathrm{~kg} / \mathrm{ha}$ de $\mathrm{N}$ mais 100 $\mathrm{kg} / \mathrm{ha}$ de $\mathrm{K}_{2} \mathrm{O}$ tiveram as melhores respostas em qualidade de armazenamento.

No que se refere à época de plantio, a formação de bulbos está relacionada à interação entre a temperatura e fotoperíodo. Nessa interação, o fator mais importante é o fotoperíodo, que determina os limites de adaptação das diferentes cultivares (Galmarini, 1997). No Nordeste, em função do fotoperíodo e ausência de precipitações, o plantio pode se realizado durante o ano todo, sendo recomendada a cultivar Alfa Tropical, cuja principal característica é sua baixa exigência em fotoperíodo, podendo ser cultivada no verão, fora da época tradicional (março/abril), para estados de Pernambuco e Bahia.

Neste trabalho, objetivou-se avaliar o efeito de doses de nitrogênio e potássio, via fertirrigação, na produtividade e armazenamento pós-colheita de bulbos de cebola, em cultivo de verão, nas condições do Vale do São Francisco.

\section{MATERIAL E MÉTODOS}

O experimento foi conduzido no período de agosto de 2001 a março de 2002, no Campo Experimental de Bebedouro, da Embrapa Semi-árido, em Petrolina-PE. O solo classificado como Latossolo Vermelho Amarelo Distróferrico (Embrapa, 1999), apresentou $\mathrm{pH}\left(\mathrm{H}_{2} \mathrm{O}\right)=6,7$; $\mathrm{Ca}=2,3 \mathrm{cmol}_{\mathrm{c}} / \mathrm{dm}^{3} ; \mathrm{Mg}=0,8 \mathrm{cmol}_{\mathrm{c}} / \mathrm{dm}^{3} ; \mathrm{Na}=0,01 \mathrm{cmol}_{\mathrm{c}} /$ $\mathrm{dm}^{3} ; \mathrm{K}=0,23 \mathrm{cmol}_{\mathrm{c}} / \mathrm{dm}^{3} ; \mathrm{Al}=0,00 \mathrm{cmol}_{\mathrm{c}} / \mathrm{dm}^{3}, \mathrm{P}($ Mehlich $)=$ $31,0 \mathrm{mg} / \mathrm{dm}^{-3}$ e M.Oे $=6,6 \mathrm{~g} / \mathrm{kg}$.

$\mathrm{O}$ delineamento experimental utilizado foi blocos ao acaso, em esquema fatorial $4 \mathrm{x} 3$, compreendendo quatro doses de nitrogênio $(0,60,120$ e $180 \mathrm{~kg} / \mathrm{ha})$ e três doses de potássio $(0,90$ e $180 \mathrm{~kg} / \mathrm{ha})$ com três repetições. As parcelas foram distanciadas uma da outra por 2,0 $\mathrm{m}$ e constituiramse de um canteiro com oito linhas de 3,0 m de comprimento, espaçadas de $0,15 \mathrm{~m}$, com $0,10 \mathrm{~m}$ entre plantas, perfazendo uma área total 3,6 m² (3,0 x 1,2 m), sendo utilizadas como área útil as seis linhas centrais, retirando-se $0,50 \mathrm{~m}$ em cada extremidade $\left(1,80 \mathrm{~m}^{2}\right)$.

A adubação de plantio constou de $60 \mathrm{~kg} / \mathrm{ha}$ de $\mathrm{P}_{2} \mathrm{O}_{5}$ (superfosfato simples) baseada na análise do solo. As adubações, nitrogenada e potássica, foram aplicadas via água de irrigação, diariamente, utilizando-se injetores hidráulicos de fertilizantes, de acordo com os diferentes tratamentos, iniciando-se aos 5 dias após o transplantio, sendo aplicadas por 44 vezes para o nitrogênio, totalizando 60 dias consecutivos e por 59 vezes para o potássio, totalizando 70 dias consecutivos. Um maior número de dias para adubação potássica deveu-se ao estádio fenológico da planta, onde o nutriente tem sua maior importância por ocasião da fase de bulbificação e início de maturação da cebola.

A semeadura da cultivar Alfa Tropical foi feita em 27 de agosto de 2001 e o transplante efetuado 35 dias após. O preparo do solo constou de aração, gradagem e levantamento dos canteiros a $0,20 \mathrm{~m}$ de altura. A cultura foi mantida no limpo por meio de capinas manuais e a irrigação por microaspersão realizada três vezes por semana, com lâminas em torno de $7 \mathrm{~mm}$, baseada na evaporação do tanque classe A, e suspensas 20 dias antes da colheita. Os demais tratos fitossanitários foram realizados de acordo com as recomendações para a cultura da cebola (Souza \& Resende, 2002).

A colheita foi realizada em 28 de dezembro de 2001, quando as plantas apresentaram sinais avançados de senescência, como amarelecimento e seca das folhas e quando mais de $70 \%$ das plantas encontravam-se estaladas. A cura foi realizada ao sol por três dias e 12 dias à sombra em galpão ventilado.

Foram avaliadas a produtividade comercial de bulbos (bulbos perfeitos e com diâmetro transversal acima de $35 \mathrm{~mm}$ ) e não comerciais (refugos) (bulbos com diâmetro inferior a $35 \mathrm{~mm}$ ) expressas em t/ha, aos 15 dias após a cura. A massa fresca de bulbo ( $\mathrm{g} / \mathrm{bulbo}$ ) foi determinada dividindo-se o massa fresca de bulbos comerciais, após a cura, pelo número de bulbos. Para avaliar a perda póscolheita, após o período de cura, os bulbos foram 
armazenados à temperatura ambiente (temperatura média mensal $26,2{ }^{\circ} \mathrm{C}$ e umidade relativa média de $66,7 \%$ ) e realizadas pesagens aos 40 e 80 dias, sendo os valores comparados àqueles obtidos ao final da cura (15 dias após colheita). Os valores foram transformados em porcentagem de perda de massa.

Os dados coletados foram submetidos à análise de variância e regressão polinomial ao nível de 5\% de probabilidade. Os dados de porcentagem foram transformados em arco-seno $\sqrt{P / 100}$ para efeitos de análise.

\section{RESULTADOS E DISCUSSÃO}

A produtividade comercial apresentou efeito quadrático na ausência da adubação potássica em função das doses de $\mathrm{N}$, com máxima produtividade na dose de 96,5 $\mathrm{kg} / \mathrm{ha}$ de N (36,5 t/ha) (Figura 1). Observou-se ainda, incremento linear na produtividade comercial com o aumento das doses de $\mathrm{N}$ quando se aplicou $90 \mathrm{~kg} / \mathrm{ha}$ de $\mathrm{K}_{2} \mathrm{O}$. Na maior dose $\left(180 \mathrm{~kg} / \mathrm{ha}\right.$ de $\left.\mathrm{K}_{2} \mathrm{O}\right)$ a resposta à adubação nitrogenada foi quadrática, com ponto de mínima produtividade na dose de $136,5 \mathrm{~kg} / \mathrm{ha}$ de nitrogênio $(31,3 \mathrm{t} /$ ha), ou seja, melhores produtividades foram obtidas com menores doses de nitrogênio, sendo a maior na ausência da adubação nitrogenada (42,1 t/ha). Portanto, isto sugere que há um nível ótimo entre as doses de $\mathrm{N}$ e $\mathrm{K}$ para se promover efeito positivo sobre a produtividade comercial da cebola.

Respostas positivas da aplicação de $\mathrm{N}$ na cultura da cebola têm sido relatadas por diversos autores até as doses de 150 kg/ha (Diaz-Perez et al., 2003; Singh et al., 2004) e $200 \mathrm{~kg} / \mathrm{ha}$ (Neeraja et al., 2001) de forma isolada. Em interação, Singh et al. (2000) verificaram maiores produtividades com $100 \mathrm{~kg} / \mathrm{ha}$ de $\mathrm{N}$ e $83 \mathrm{~kg} / \mathrm{ha}$ de $\mathrm{K}_{2} \mathrm{O}$, respectivamente; Syed et al. (2000) com $120 \mathrm{~kg} / \mathrm{ha}$ de N e $90 \mathrm{~kg} / \mathrm{ha}$ de $\mathrm{K}_{2} \mathrm{O}$; enquanto Mandira \& Khan (2003), obtiveram melhores rendimentos com $150 \mathrm{~kg} / \mathrm{ha}$ de $\mathrm{N}$ e 75 $\mathrm{kg} / \mathrm{ha}$ de $\mathrm{K}_{2} \mathrm{O}$, respectivamente.

A literatura relata um antagonismo entre esses elementos, no entanto, os resultados obtidos sugerem uma resposta complementar, com o $\mathrm{N}$ como principal fator que afeta positivamente a produtividade. De acordo com Silva Junior (1987) esse antagonismo tem origem em adubações desequilibradas, afirmação corroborada por Resende et al. (1997), que afirmam que há um efeito significativo e complementar na absorção de $\mathrm{N}$ e K, e que o importante é a necessidade de um adequado nível de $\mathrm{K}$ para incrementar a produtividade com a adição de $\mathrm{N}$.

A produtividade não comercial (refugos) foi reduzida de forma linear na ausência da adubação potássica, assim como nas doses de 90 e $180 \mathrm{~kg} / \mathrm{ha}$ de $\mathrm{K}_{2} \mathrm{O}$, com o incremento das doses de $\mathrm{N}$. Na ausência das adubações nitrogenadas e potássicas ocorreram maiores produções de bulbos refugos que foram reduzidos quando da aplicação de doses crescentes desses nutrientes (Figura 2). May (2006) encontrou com aplicação de $115 \mathrm{~kg} / \mathrm{ha}$ de $\mathrm{N}$ e 150 $\mathrm{kg} / \mathrm{ha}$ de $\mathrm{K}_{2} \mathrm{O}$, reduções na quantidade de bulbos refugos. Ghaffoor et al. (2003), constataram menores produtividades de refugos com o aumento na adubação com NPK. Esses autores demonstraram também a capacidade de resposta

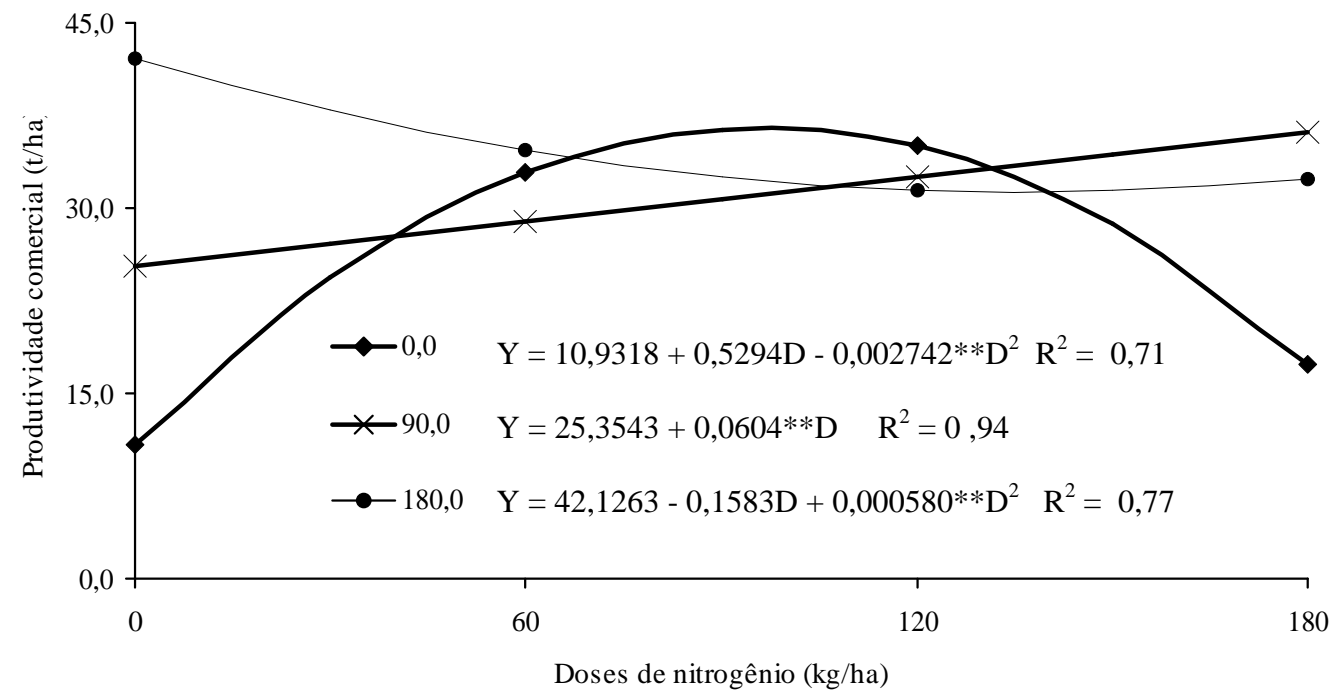

Figura 1 - Produtividade comercial de bulbos de cebola nas doses 0; 90 e $180 \mathrm{~kg} / \mathrm{ha}$ de $\mathrm{K}_{2} \mathrm{O}$ em função das doses de $\mathrm{N}$. Petrolina-PE, 2001/2002. 
da cebola às aplicações de N e K. Lee et al. (2003) e Mandira \& Khan (2003), também relataram que estes elementos contribuem marcadamente para uma melhor produtividade da cultura, sobretudo na produção de bulbos de maior tamanho.

A produção de massa fresca dos bulbos na ausência da adubação potássica e com a dose de $90 \mathrm{~kg} / \mathrm{ha}$ apresentou efeito linear positivo em função das doses de N. Para a maior dose de K (180 kg/ha de $\left.\mathrm{K}_{2} \mathrm{O}\right)$ observou-se redução linear gradativa com o incremento das doses de $\mathrm{N}$ (Figura 3). Esses resultados, assim como os anteriores, sugerem uma resposta positiva e complementar da interação entre estes nutrientes, quando o equilíbrio de doses é o fator mais relevante dessa interação. May et al. (2007) afirmaram ser a aplicação de nitrogênio mais expressiva em termos de relevância que a resposta à aplicação da adubação potássica. Também em interação, Singh et al. (2004) e Dilruba et al. (2006) observaram incrementos na massa fresca do bulbo nas doses de $150 \mathrm{e}$ $120 \mathrm{~kg} / \mathrm{ha}$ de $\mathrm{N}$ com $120 \mathrm{~kg} / \mathrm{ha}$ de $\mathrm{K}_{2} \mathrm{O}$.

A conservação pós-colheita apresentou resultados diferenciados das doses de $\mathrm{N}$ e $\mathrm{K}$ em função do período de armazenagem. Aos 40 dias de armazenamento, não se constataram efeitos significativos das doses de $\mathrm{N}$ e K. Observaram-se variações para doses de $\mathrm{N}$ entre 10,6 e $13,4 \%$ e para doses de K entre 11,0 e 13,7 $\%$. No entanto, aos 80 após cura, houve efeito significativo dos tratamentos com efeito quadrático para as regressões (Figura 4). O desdobramento da interação N x K revelou na ausência da adubação potássica ponto de mínima perda na dose de $92 \mathrm{~kg} /$ ha de $\mathrm{N}(25,9 \%)$. Para as doses de 90 e $180 \mathrm{~kg} / \mathrm{ha}$ de $\mathrm{K}_{2} \mathrm{O}$ obtiveram-se respostas quadráticas, que permitiram estimas a doses de 107,4 e $125,6 \mathrm{~kg} / \mathrm{ha}$ de $\mathrm{N}$, como as que promoveram as maiores percentagens de perdas de massa de bulbos comerciais, 27,7 e $30,0 \%$, respectivamente (Figura 4).

A maior conservação pós-colheita apresenta relevante importância por ocasião da comercialização do produto, que apresenta grandes variações de preços, em curtos períodos de tempo. O produtor em função do conhecimento das perdas de massa da cebola poderá alcançar melhores cotações de preços e incrementar ou maximizar seus lucros, em função da volatilidade do mercado ceboleiro. Os resultados obtidos em póscolheita são comparáveis aos encontrados por Singh \& Dhankhar (1991), que verificaram em bulbos armazenados sob condições ambientais que a perda de massa e a incidência de bulbos podres e brotados aumentaram com o incremento das doses de nitrogênio (80 a $160 \mathrm{~kg} / \mathrm{ha}$ de $\mathrm{N}$ ) e reduziram com a dose de $100 \mathrm{~kg} /$ ha de $\mathrm{K}_{2} \mathrm{O}$. Segundo esses autores a dose de $80 \mathrm{~kg} / \mathrm{ha}$ de $\mathrm{N}$ mais $100 \mathrm{~kg} / \mathrm{ha}$ de $\mathrm{K}_{2} \mathrm{O}$ obtiveram as melhores respostas em termos de armazenamento. Enquanto, Hussaini et al. (2000) não observaram efeitos do nitrogênio até a dose de $164 \mathrm{~kg} / \mathrm{ha}$, dezenove semanas após a colheita.

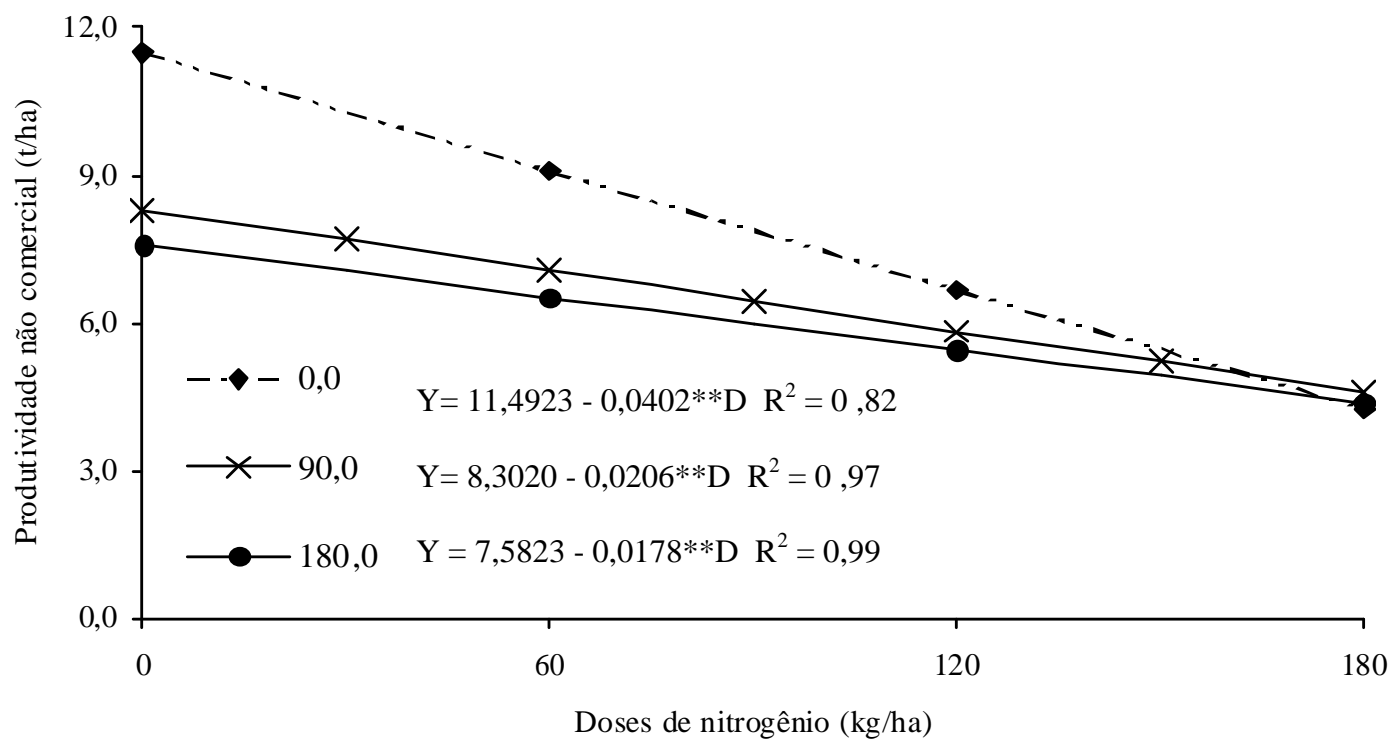

Figura 2 - Produtividade não comercial (refugos) de bulbos de cebola nas doses 0; 90 e $180 \mathrm{~kg} / \mathrm{ha}$ de $\mathrm{K}_{2} \mathrm{O}$ em função das doses de N. Petrolina-PE, 2001/2002. 


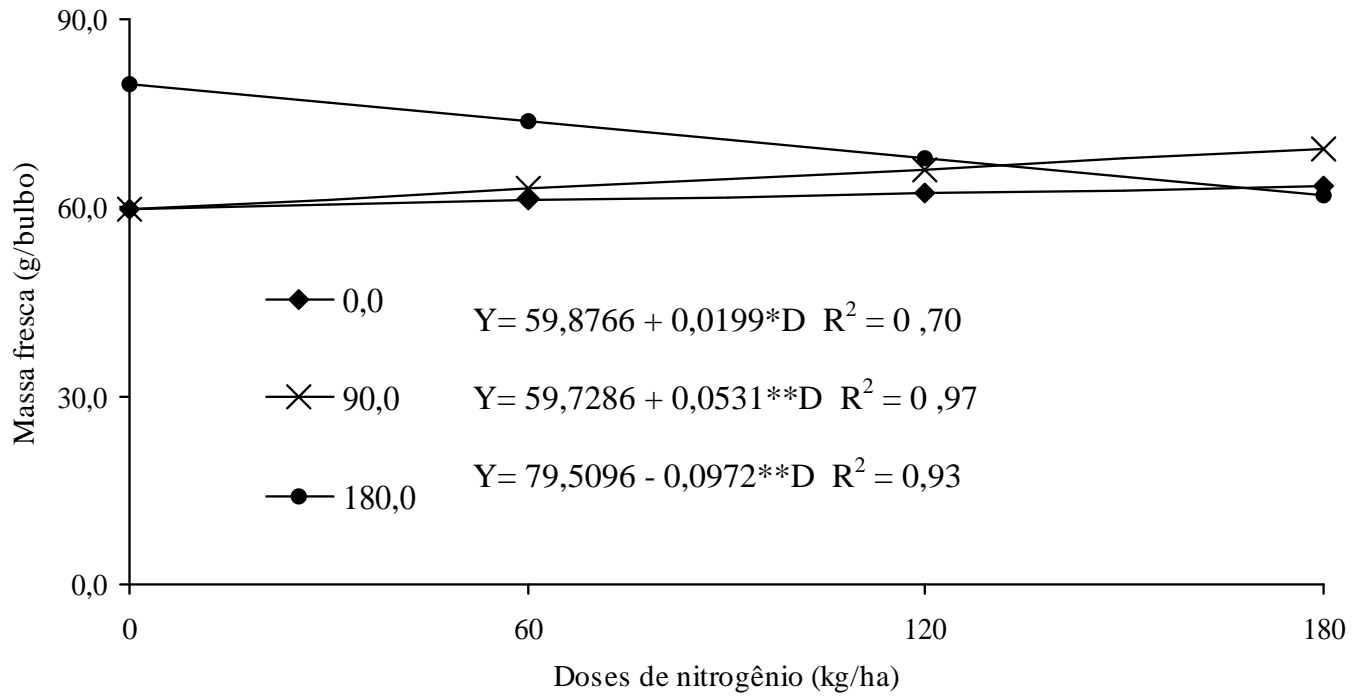

Figura 3 - Massa fresca de bulbos de cebola nas doses 0; 90 e 180 kg/ha de $\mathrm{K}_{2} \mathrm{O}$ em função das doses de N. PetrolinaPE, 2001/2002.

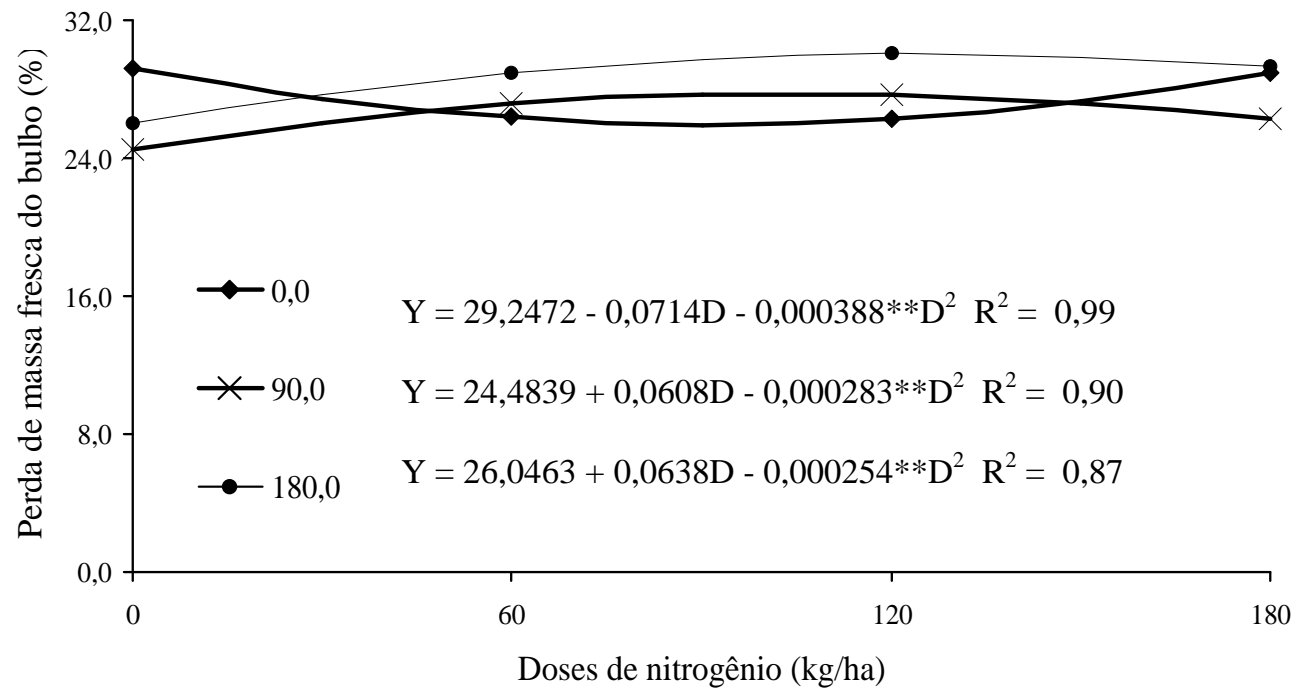

Figura 4 - Perda de massa fresca de bulbo aos 80 dias após cura nas doses 0; 90 e 180 kg/ha de $\mathrm{K}_{2} \mathrm{O}$ em função das doses de N. Petrolina-PE, 2001/2002.

\section{CONCLUSÕES}

A produtividade comercial apresentou efeito linear para a dose intermediária de $\mathrm{N}$ e quadrático na ausência e maior dose da adubação potássica.

A aplicação de $\mathrm{N}$ e $\mathrm{K}$ reduziram a produtividade não comercial de bulbos (refugos).

Há um efeito significativo e complementar na absorção de $\mathrm{N}$ e K, em termos produtivos e conservação pós-colheita, onde o $\mathrm{N}$ é o nutriente de maior importância na interação.

\section{REFERÊNCIAS BIBLIOGRÁFICAS}

AKHTAR, M.E.; BASHIR, K.; KHAN, M.Z.; KHOKLAR, K.M. Effect of potash application on yield of different varieties of onion (Allium cepa L.). Asian Journal of

Plant Science, Islamabad, v.1, n.4, p.324-325, 2002. 
BATAL, K.M.; BONDARI, K.; GRANBERRY, D.M.; MULLINIX, B.G. Effects of source, rate, and frequency of $\mathrm{N}$ application on yield, marketable and rot incidence of sweet onion (Allium cepa L. cv. Granex-33). Journal Horticultural Science, Ashford, v.69, n.6, p.1043-1051, 1994.

BOYHAN, G.E.; TORRANCE, R.L.; HILL, C.R. Effects of nitrogen, phosphorus, and potassium rates and fertilizer sources on yield and leaf nutrient status of short-day onions. HortScience, Alexandria, v.42, n.3, p.653-660, 2007.

DIAZ-PEREZ, J.C.; PURVIS, A.C.; PAULK, J.T. Bolting, yield, and bulb decay of sweet onion as affected by nitrogen fertilization. Journal American Society

Horticultural Science, Alexandria, v.128, n.1, p.144-149, 2003.

DILRUBA, S.; ALAM, M.M.; RAHMAN, M.A.; HASAN, M.F. influence of nitrogen and potassium on yield contributing bulbs traits of onion. International Journal of Agricultural Research, Nairobi, v.1, n.1, p.85-90, 2006.

EMPRESA BRASILEIRA DE PESQUISA AGROPECUÁRIA. Sistema brasileiro de classificação de solos. Brasília, DF, 1999. 412p.

FARIAS, C.M. de; PEREIRA, J.R. Fontes e níveis de nitrogênio na produtividade da cebola no Vale do São Francisco. Pesquisa Agropecuária Brasileira, Brasília, v.27, n.3, p.403-407, mar. 1992.

FILGUEIRA, F.A.R. Novo manual de olericultura: agrotecnologia moderna produção e comercialização de hortaliças. 3.ed. Viçosa, MG: UFV, 2008. 421p.

FOOD AND AGRICULTURE ORGANIZATION.

Agricultural production, primary crops. Disponível em: Shttp://Www.fao.orgi . Acesso em: 1 fev. 2008.

GALMARINI, C.R. Caracteristicas botanicas y fisiologicas. In: __ . Manual del cultivo de la cebolla. San Juan: INTA, 1997. 128p.

GHAFFOOR, A.; JILANI, M.S.; KHALIQ, G.; WASEEM, K. Effect of different NPK levels on the yield of three onion (Allium cepa L.) varieties. Asian Journal Plant Sciences, Islamabad, v.2, n.3, p.342-346, 2003.
HUSSAINI, M.A.; AMANS, E.B.; RAMALAN, A.A. Yield, bulb size distribution, and storability of onion (Allium cepa L.) under different levels of $\mathrm{N}$ fertilization and irrigation regime. Tropical Agriculture, Saint Augustine, v.77, n.3, p.145-149, 2000.

\section{INSTITUTO BRASILEIRO DE GEOGRAFIA E}

ESTATÍSTICA.Produção agrícola municipal. Disponível em: <http://www.sidra.ibge.gov.bri $>$. Acesso em: 1 fev. 2008.

LEE, J.T.; HA, I.J.; LEE, C.J.; MOON, J.S.; CHO, Y.C. Effect of $\mathrm{N}, \mathrm{P}_{2} \mathrm{O}_{5}$, and $\mathrm{K}_{2} \mathrm{O}$ application rates and top dressing time on growth and yield of onion (Allium cepa L.) under spring culture in low land. Korean Journal Horticultural Science and Technology, Suwon, v.21, n.4, p.260-266, 2003.

MAGALHÃES, J.R. Nutrição e adubação da cebola. In: SIMPÓSIO SOBRE NUTRIÇÃO E ADUBAÇÃO DE HORTALIÇAS, 1990, Jaboticabal. Anais... Piracicaba: Potafos, 1993. p.381-399.

MANDIRA, C.; KHAN, A.H. Effect of nitrogen and potassium on growth, yield and yield attributes of onion. New Agriculturist, Allahabad, v.14, n.1/2, p.911, 2003.

MAY, A. Desempenho de híbridos de cebola em função da população de plantas e fertilização nitrogenada e potássica. 2006. 142f. Tese (Doutorado em Produção Vegetal)-Universidade Estadual Paulista, Jaboticabal, 2006.

MAY, A.; CECÍLIO FILHO, A.B.; PORTO, D.R.Q.; VARGAS, P.F.; BARBOSA, C.B. Produtividade de híbridos de cebola em função da população de plantas e da fertilização nitrogenada e potássica. Horticultura Brasileira, Brasília, v.25, n.1, p.53-59, jan./mar. 2007.

MOHANTY, B.B.; DAS, J.N. Response of rabi onion cv. Nasik Red to nitrogen and potassium fertilization. Vegetable Science, Varanasi, v.28, n.1, p.40-42, 2001.

NEERAJA, G.; REDDY, K.M.; REDDY, M.S.; RAO, V.P. Influence of irrigation and nitrogen levels on bulb yield, nutrient uptake an nitrogen use efficiencies in rabi onion (Allium cepa). Indian Journal Agricultural Sciences, New Delhi, v.7, n.2, p.109-111, 2001. 
PÔRTO, D.R. de Q.; CECILIO FILHO, A.B.; MAY, A.; VARGAS, P.F. Acúmulo de macronutrientes pela cultivar de cebola "Superex" estabelecida por semeadura direta. Ciência Rural, Santa Maria, v.37, n.4, p.949-955, abr. 2007.

RESENDE, G.M. de; SILVA, G.L. da; PAIVA, L.E.; DIAS, P.F.; CARVALHO, J.G. de. Resposta do milho (Zea mays L.) a doses de nitrogênio e potássio em solo da região de Lavras-MG. II. Macronutrientes na parte aérea. Ciência e Agrotecnologia, Lavras, v.21, n.1, p.477-483, jan./mar. 1997.

SHARMA, R.P. Effect of planting material, nitrogen and potash on bulb yield of rainy-season onion (Allium серa). Indian Journal of Agronomy, New Delhi, v.37, n.4, p.868-869, 1992

SHUKLA, V.; RAO, K.P.G.K.; PRABHAKAR, B.S. Effect of nitrogen on bulb yield and keeping quality of onion cultivars. Progressive Horticulture, Uttar Pradesh, v.21, n.3/4, p.244-245, 1992.

SILVA JUNIOR, A.A. Adubação mineral e orgânica em repolho: II., concentração de nutrientes na folha $\mathrm{e}$ precocidade. Horticultura Brasileira, Brasília, v.5, n.1, p.15-17, maio 1987.

SINGH, D.; SHARMA, R.P. Effect of soil moisture regimes and nitrogen fertilization on onion. Indian Journal of Agronomy, New Delhi, v.36, n.1, p.125-126, 1991.

SINGH, J.; DHANKHAR, B.S. Effect of nitrogen, potash and zinc on storage loss of onion bulbs (Allium cepa L.).
Vegetable Science, Varanasi, v.18, n.1, p.16-23, 1991.

SINGH, R.P.; JAIN, N.K.; POONIA, B.L. Response of Kharif onion to nitrogen, phosphorus and potash in eastern plains of Rajasthan. Indian Journal Agricultural Science, New Delhi, v.70, n.3, p.871-872, 2000.

SINGH, S.; YADAV, P.K.; SINGH, B. Effect of nitrogen and potassium on growth and yield of onion (Allium cepa L.) cv. Pusa Red. Haryana Journal Horticultural Sciences, Hisar, v.33, n.3/4, p.308-309, 2004.

SOUZA, R.J.; RESENDE, G.M. de. Cultura da cebola. Lavras: UFLA, 2002. 115p. (Textos Acadêmicos -

Olericultura, 21).

SYED, N.; MUNIR, M.; ALIZAI, A.A.; GHAFOOR, A. Onion yield and yield components as function of the levels of nitrogen and potassium application. Pakistan Journal Biological Sciences, Faisalabad, v.3, n.12, p.2069-2071, 2000.

VACHCHANI, M.U.; PATEL, Z.G. Growth and yield of onion (Allium cepa L.) as influenced by levels of nitrogen, phosphorus and potash under South Gujarat conditions. Progressive Horticulture, Uttar Pradesh, v.25, n.3/4, p.166-167, 1996.

VIDIGAL, S.M. Adubação nitrogenada de cebola irrigada cultivada no verão: projeto Jaíba, Norte e Minas Gerais. 2000. 136f. Tese (Doutorado em Produção Vegetal)-Universidade Federal de Viçosa, Viçosa, MG, 2000. 